\title{
Performance Modeling of IEEE 802.15.4-TSCH with Shared Access and ON-OFF traffic
}

\author{
Sahar Ben Yaala \\ Innov'COM Laboratory, Sup'Com \\ University of Carthage, Tunisia \\ email: benyaala@unistra.fr
}

\author{
Fabrice Théoleyre \\ ICube Laboratory \\ CNRS, University of Strasbourg, France \\ email: theoleyre@unistra.fr
}

\author{
Ridha Bouallegue \\ Innov'COM Laboratory, Sup'Com \\ University of Carthage, Tunisia \\ email: ridha.bouallegued supcom.tn
}

\begin{abstract}
Many applications for the Internet of Things require strict guarantees to operate properly. IEEE802.15.4-TSCH has been proposed to design a real-time access for industrial wireless networks. The standard relies on a strict schedule of the transmissions., and combines frequency hopping to provide highreliability. While most of the propositions focus on a periodic traffic, we propose here to address the non-periodic case. In particular, shared access would be more efficient to reduce the power duty cycle by multiplexing the transmissions through the same cells. We propose here a Markov model for a shared access and sporadic traffic. By formulating the collision probability, the scheduler can decide how many transmitters to assign to a given shared cell according to the amount of traffic each node generates on average. The simulations validate the accuracy of our model to capture the collision rate for non-periodic traffic.
\end{abstract}

Index Terms-IEEE802.15.4-TSCH; Industrial IoT; Markov Chain; on-off traffic; shared access

\section{INTRODUCTION}

Industry 4.0 relies extensively on the Internet of Things (IIoT) paradigm [1]. In this context, the IEEE802.15.4-TSCH (Time Slotted Channel Hopping) standard targets specifically applications with high reliability and a low delay.

IEEE802.15.4-TSCH relies on a strict schedule of the transmissions into cells, organized into a scheduling matrix. A transmitter may use shared cells, with a random access. In this situation, the same cell is allocated to multiple transmitters which use all slotted Aloha combined with a random backoff when a collision is detected. Shared cells are typically used for broadcast packets, forcing all the nodes to stay awake in the corresponding cells to receive them.

Assigning the bandwidth (i.e. cells) was extensively studied in the past [2]. Both centralized and distributed scheduling approaches have been proposed. Only Elsts et al. have proposed to exploit both shared and dedicated cells to handle unpredictable traffic [3]. However, they focus on the scheduling algorithm and evaluate its performance with simulations.

Unfortunately, some applications may generate non-periodic traffic, particularly in smart cities [4]. For instance, a surveillance application generates a burst of data packets when an intruder/object is detected. Smart parking applications exhibit similarly an event-triggered traffic pattern: packets are sporadically generated when a parking place is released.

The performance of multichannel Aloha and compressive sensing techniques have already been studied in the past [5].
We aim here to focus on the comparison of shared versus dedicated cells in the schedule of TSCH. Using only dedicated cells would prevent the collisions, but one dedicated cell is required for each radio link, whatever the traffic volume is. With sporadic traffic, a receiver has to wake-up in each dedicated cell to sense the medium during a given time offset, whenever the cell is busy or not.

The random access during the shared cells in TSCH has already been modeled with Markov Chains (e.g. [6], [7]). However, to the best of our knowledge, the behavior of TSCH with bursty traffic and shared cells has not yet been investigated.

The contributions of this paper are threefold:

1) we provide an analytical model to describe the behavior of a IEEE802.15.4-TSCH network. We consider both on-off traffic, and the usage of shared cells. We analyze the evolution of the queue size for on-off traffic, and the mutual impact of the different transmitters;

2) we study the performance of the random access of IEEE802.15.4-TSCH in term of throughput and delay with sporadic traffic;

3) we validate our models with simulations to highlight the accuracy of our model.

\section{RELATED WORK}

We present here the main mechanisms of the IEEE 802.15.4-TSCH standard, and how it it provides both a contention-free and a random-access mechanism. Then, we will detail specifically the random access mode, and how it was modeled in the literature.

\section{A. IEEE 802.15.4-TSCH}

IEEE 802.15.4-2015 has proposed the TimeSlotted Channel Hopping (TSCH) mode, which relies on a strict schedule of the transmissions. The slotframe contains a fixed number of timeslots, during which at most one frame and its acknowledgment are transmitted. Each timeslot is labelled with an Absolute Sequence Number (ASN) which counts the number of timeslots since the PAN coordinator started. Based on the schedule, a node can decide its role (transmitter/receiver/sleeping mode) at the beginning of each timeslot.

IEEE 802.15.4-2015 TSCH implements a channel hopping approach to combat external interference and signal fading 


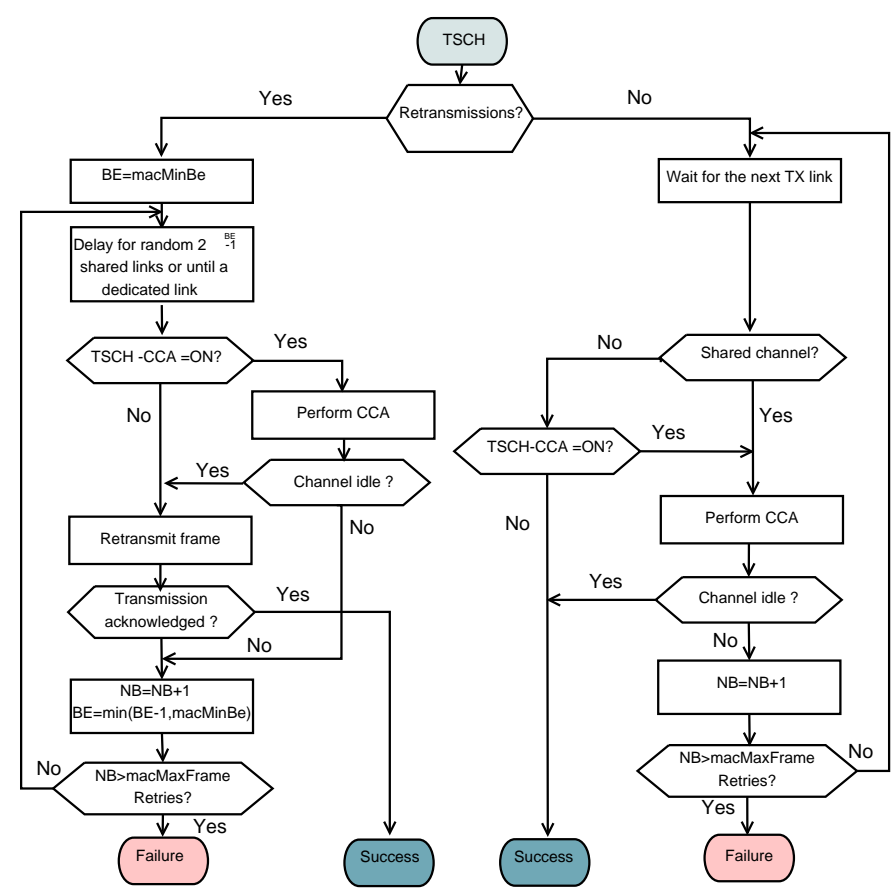

Fig. 1: State Machine of $\mathrm{TSCH}$ for the random access mode [9].

and, thus, to achieve high network reliability [8]. For this purpose, each cell in the schedule is defined by a pair of timeslot and channel offset. At the beginning of each timeslot, the actual frequency to use is derived from the channel offset and the ASN.

\section{B. TSCH CSMA-CA Algorithm}

The contention mechanism depends on the cell's type in the schedule. If the cell is dedicated, the transmitter has just to transmit its frame without contention, after a fixed offset from the beginning of the timeslot to deal with clock drifts. In a shared cell, several transmitters may be assigned to the same timeslot, leading possibly to collisions. Thus, TSCH has defined a specific random access method (Fig. 1). The process depends on the packet's type:

broadcast: if the packet is transmitted in broadcast, the transmitter just dequeues the packet, and transmits it directly during the next dedicated cell. The method is similar for packets without acknowledgement;

acknowledged unicast: the first copy is transmitted without contention (as for broadcast). If no ack is received, the transmitter assumes a collision occurred. It selects randomly a backoff value, and has to wait the corresponding number of shared cells. The CCA at the beginning of the slot is consequently used only to detect external interference.

Let us consider the scenario depicted in Figure 2. During the first shared cell, only S1 transmits a packet, which is correctly received and acknowledged by D. Then, both nodes S1 and S2 generate a packet. Since no backoff is triggered for the first

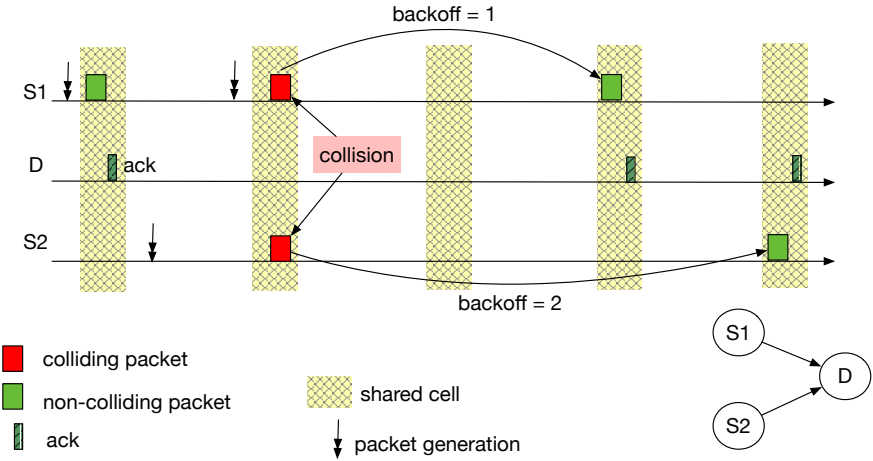

Fig. 2: Transmission which may occur within a shared cell in TSCH.

transmission, they both try to submit a packet during the next shared cell, and cause a collision. Since they don't receive any ack from $\mathrm{D}$, they choose a random backoff (respectively 1 and 2). S1 will consequently transmit in the second next shared cell, and S2 in the third one.

A node picks its backoff between 0 and $2^{B E}-1$ (the default initial $\mathrm{BE}$ value is equal to 2), the backoff exponent being incremented after each unsuccessful transmission. However, a backoff is only used for the retransmissions. For all these reasons, the collisions cannot be neglected and may impact negatively the reliability.

\section{Modeling}

The TSCH mode of IEEE802.15.4 has already been studied in the past. Bianchi has proposed the first model for IEEE802.11-DCF networks to represent the behavior of a random backoff based MAC [10]. They provide a Markov model to evaluate the performance of IEEE802.11 when the radio channel is ideal (i.e. without errors). This model has been extended to deal with a variable backoff window [11]. These models consider that a transmitter has always a packet to transmit (saturated mode).

Misic et al. [12] have extended this work to model a IEEE802.15.4 network, with a M/G/1 queueing system. They studied the impact of a different frame length, and the channel sensing mechanism.

Recently, De Guglielmo et al. [13] proposed the first model for TSCH-CSMA mode. They also consider the capture effect, where a transmission may be received even if another (weaker) transmission overlaps.

In this paper, we focus on the TSCH mode of IEEE802.15.4. We aim to consider the non saturated mode, which seems more realistic for many industrial low-power applications.

\section{TSCH-CSMA/CA MODEL}

We aim here to propose an analytical model to represent the shared access in TSCH. We model each device by a queue, and we reference all the possible transitions (retransmissions, success) depending on the network characteristics (e.g. number of transmitters, queue states of the interfering nodes). 


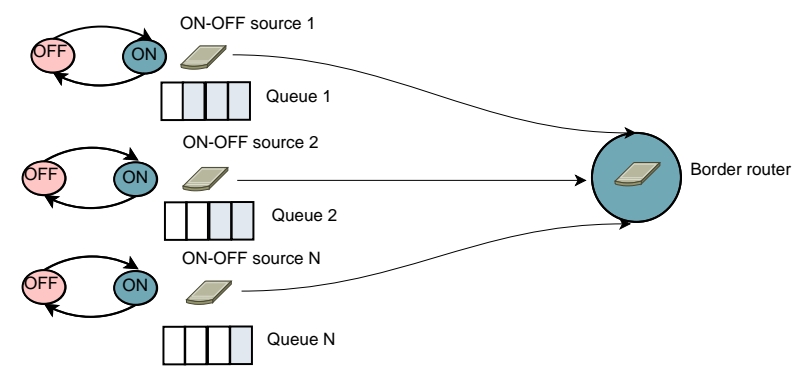

Fig. 3: Topology considered in the model.

TABLE I: Notation packets) in a given slot. We denote by $\mathcal{A}_{i}$ the arrival process of $i$ packets into the queue since the last shared cell and by $P_{s}$ the probability to transmit a packet successfully.

Each state in the model represents the number of enqueued packets for the corresponding source node. At the $i^{t h}$ shared cell, the queue of the source node will change according to (i) the number of packets generated by the node, (ii) the success of the last transmission, in the previous shared cell.

More precisely, we compute the queue size at the beginning of each shared cell. We denote by $P_{\text {queue }}[i \rightarrow j]$ the probability to pass from $\mathrm{i}$ to $\mathrm{j}$ packets in the queue between two consecutive shared cells. When the queue was empty in the last shared cell, $i$ packets may have been generated meanwhile, leading to:

$$
P_{\text {queue }}[0 \rightarrow i]=\mathcal{A}_{i}, i \in[0, L]
$$

Inversely, if the queue was not empty, the result depends on the success of the previous transmission: if the last transmission has succeeded, one packet has been removed from the queue (first term of the equation), else, the packet stayed in the queue (second term). Besides, we have also to consider

Binom The distribution of active nodes the amount of packets generated meanwhile:

$$
\begin{aligned}
P_{\text {queue }}[i \rightarrow j]= & P_{s} \cdot \mathcal{A}_{j-i+1}+\left(1-P_{s}\right) \cdot \mathcal{A}_{j-i} \\
& j \geq i \wedge i \in[1, L-1] \wedge j \in[1, L-1]
\end{aligned}
$$

The number of packets in the queue may also decrease if the last packet was successfully transmitted and there are no arrival:

$$
P_{\text {queue }}[i \rightarrow j]=P_{s} \cdot \mathcal{A}_{0}, j=i-1 \wedge i \in[1, L]
$$

\section{B. Number of contenders}

In order to model the network, we have to compute the evolution of the number of transmitters at each slot. Obviously, a larger number of transmitters also increases the collision probability. Each state in the model represents the number of active nodes (with a non null queue size) that share a given slot.

We assume that each node corresponds to an $\mathrm{ON}-\mathrm{OFF}$ source connected to the border router, as illustrated in Figure 3. $\mathrm{ON}$ and OFF durations are exponentially distributed (in our simulations with an average rate of 0.4 and 0.6 respectively). During the ON period, the packets are generated following a poisson process with the corresponding rate $\lambda$ (Poison traffic). In OFF state, no traffic is generated.

Let's assume that all the nodes are initially inactive. A node becomes active when it has generated at least one packet, and has consequently a non empty queue. Since all the nodes have the same traffic process, the number of active nodes follows a binomial distribution Binom:

$$
\begin{aligned}
P_{a c t}[0 \rightarrow n] & =\operatorname{Binom}(n, N), n \in[0, N] \\
& =\left(\begin{array}{c}
N \\
n
\end{array}\right) \mathcal{A}_{1}^{n}\left(1-\mathcal{A}_{1}\right)^{(N-n)}
\end{aligned}
$$

Let us now consider the case with initially $n$ already active nodes. A node becomes inactive if it has only one packet in the impact of a bursty traffic on TSCH. Thus, we refer to a specific node and we focus on the size of is queue (number of 
the queue, succeeds to transmit it, and no packet has been generated meanwhile. To formulate this probability, we present next a multidimensional Markov chain.

\section{DTMC MODEL FOR THE NUMBER OF ACTIVE NODES}

We define now a multidimensional discrete time Markov chain (DTMC) to represent the number of active nodes at a given instant. Each state in the DTMC is a 4-uplet $(m, i, r, w)$, where $m$ is the number of active nodes $(m<N), i$ is the number of packets in the queue $(i \leq L), r$ is the value of the retransmission counter $\left(r \leq \max _{R}\right)$ and $\mathrm{w}$ is the value of the backoff window counter $\left(w \leq W_{\max }\right)$.

We first formulate the different transitions in the DTMC. Then, after having defined the transition probabilities, we derive the transition matrix of the DTMC $\mathcal{M}$.

Since the Markov chain is irreducible and aperiodic, we have to resolve the following set of equations to obtain the stationary distribution $\pi$ :

$$
\begin{aligned}
& \sum \pi=1 \\
& \pi \cdot \mathcal{M}=\pi
\end{aligned}
$$

We can note that $\pi$ depends on the 4-uplet $(m, i, r, w)$, and that $\pi$ and $\mathcal{M}$ are both dependent on the success rate of the packets transmissions.

In the next part, we formulate the elements of the transition matrix $\mathcal{M}$. In particular, we refer to a specific node to find out the number of enqueued packets. We make a distinction between the three cases:

1) the number of active nodes increases;

2) the number of active nodes decreases;

3) the number of active nodes remains unchanged.

\section{A. Case1: the number of active nodes increases}

Let denote by $P_{s_{1} \rightarrow s_{2}}$ the probability to pass from the state $s_{1}$ to the state $s_{2}$ in the Markov chain.

1) Case 1.1: the number of packets in the queue of the source node increases.

If the source node transmits its packet with success during the first attempt, the packet will leave the queue. The source node transmits successfully its packet and receives $(j-i+1)$ new packets. It contends with the other $(m-1)$ active nodes, $(n-m)$ other nodes generated one packet and became active. Finally:

$$
\begin{array}{r}
P_{(m, i, 0,0) \rightarrow(n, j, 0,0)}=P_{s}[m] \cdot \operatorname{Binom}(n-m, N-m) \cdot \mathcal{A}_{j-i+1} \\
n>=m, j>i \quad(8)
\end{array}
$$

If the transmission has on the contrary failed, the corresponding packet has to be retransmitted, and must also be considered for the queue size:

$$
\begin{aligned}
& P_{(m, i, r, w) \rightarrow(n, j, r+1, w)}= \\
& \quad\left(1-P_{s}[m]\right) \cdot \operatorname{Binom}(n-m, N-m) \cdot \mathcal{A}_{j-i} \\
& n \geq m, j>i
\end{aligned}
$$

2) Case 1.2: The number of packets in the queue of the source node decreases.

The queue size can decrease only if the packet was correctly transmitted, and the source node doesn't generate another packet meanwhile. The number of transmitters may increase because $(n-m)$ among the $(N-m)$ inactive nodes will change their state:

$$
P_{(m, i, 0,0) \rightarrow(n, i-1,0,0)}=P_{s}[m] \cdot \operatorname{Binom}(n-m, N-m) \cdot \mathcal{A}_{0}
$$

3) Case 1.3: The number of packets in the queue of the source node remains unchanged.

The queue size remains unchanged in two situations. If the source node has correctly transmitted its previous packet, a new one has been generated:

$$
\begin{aligned}
& P_{(m, i, 0,0) \rightarrow(n, j, 0,0)}= \\
& \quad P_{s}[m] \cdot \operatorname{Binom}(n-m, N-m) \cdot \mathcal{A}_{1} n \geq m, j=i
\end{aligned}
$$

If inversely the previous transmission has failed, no other packet has been generated:

$$
\begin{aligned}
& P_{(m, i, r, w) \rightarrow(n, j, r+1, w)}= \\
& \left(1-P_{s}[m]\right) \cdot \operatorname{Binom}(n-m, N-m) \cdot \mathcal{A}_{0} \\
& n \geq m, j=i
\end{aligned}
$$

\section{B. Case 2: the number of active nodes decreases}

At most one node can become inactive: its transmission was successful, and it didn't generate another packet. Since no collision occurred, this means that the other nodes stay active, and cannot reduce their queue size.

Thus, $(N-m)$ inactive nodes do not change their state, and the number of packets in the queue of the source node decreases:

$$
\begin{array}{r}
P_{(m, i, 0,0) \rightarrow(n, j, 0,0)}=P_{s}[m] \cdot \operatorname{Binom}(0, N-m) \cdot \mathcal{A}_{0} \\
m>n, i=1, j=i-1
\end{array}
$$

\section{Case 3: the number of active nodes remains unchanged}

We can make a distinction between two cases, depending on the generation of another packet meanwhile, or not.

1) Case 3.1: the number of packets in the queue of the source node remains unchanged (collision without new packet generation):

$$
\begin{array}{r}
P_{(m, i, r, w) \rightarrow(n, j, r+1, w)}=\left(1-P_{s}[m]\right) \cdot \operatorname{Binom}(0, N-m) \cdot \mathcal{A}_{0} \\
m=n, j=i \quad(14)
\end{array}
$$

2) Case 3.2: the number of packets in the queue of the source node remains unchanged (successful transmission with new packet generation):

$$
\begin{gathered}
P_{(m, i, r, w) \rightarrow(n, j, r, w)}=\left(P_{s}[m]\right) \cdot \operatorname{Binom}(0, N-m) \cdot \mathcal{A}_{1} \\
m=n, j=i
\end{gathered}
$$


3) Case 3.3: the number of packets in the queue of the source node increases:

$$
\begin{aligned}
& P_{(m, i, r, w) \rightarrow(n, j, r+1, w)}= \\
& \left(1-P_{s}[m]\right) \cdot \operatorname{Binom}(0, N-m) \cdot \mathcal{A}_{1} \\
& m=n, j>i
\end{aligned}
$$

\section{Performance Evaluation}

We study here the ability of the system to deal with bursty arrivals. In particular, we analyse the impact of the number of transmitters on the reliability.

Since the Markov chain is aperiodic and irreducible, we apply the normalization methods as described previously. Once the steady state distribution is determined, we can compute our performance indicators. We implement our simulator in matlab. The number of transmitters is variable between 2 and 30 . We pick ON and OFF durations from exponential distributions with average rates 0.4 and 0.6 respectively. During ON state, we suppose a poisson traffic with rate 0.5 . We report results over 1000 time intervals.

\section{A. Collision probability}

We investigate first the impact of the number of transmitters on the number of collisions. We formulate the collision probability as follows:

$$
\begin{gathered}
P_{c}=\frac{n b \text { coll. }}{n b . \text { txed pkts }} \\
\text { nb coll. }=\sum_{m=0}^{N} \sum_{i=0}^{L} \sum_{r=0}^{\max _{R}} \sum_{w=0}^{W_{\max }}\left(1-P_{s}[m]\right) \cdot \pi(m, i, r, w) \\
\text { nb. txed pkts }=\sum_{m=0}^{N} \sum_{i=0}^{L} P_{s}[m] \cdot \pi(m, i, r, w) \\
+\sum_{m=0}^{N} \sum_{i=0}^{L} \sum_{r=0}^{m^{m_{2}}} \sum_{w=0}^{W_{\max }}\left(1-P_{s}[m]\right) \cdot \pi(m, i, r, w)
\end{gathered}
$$

When many nodes contend for a shared cell, the probability of collision increases (Fig. 4). We can see that analytical and simulation results are very close to each other. We can also remark that the collision probability is very high for the shared cells: the probability to have several active nodes is high, and the backoff mechanism of TSCH is quite agressive. A bursty traffic means also the packets are not uniformly distributed among the different slotframes, increasing the collision probability.

\section{B. Throughput}

We define the throughput as the amount of successfully delivered data packets per node:

$$
\begin{aligned}
& \text { Throughput }= \\
& \sum_{m=0}^{N} \sum_{i=0}^{L} \sum_{r=0}^{\max _{R}} \sum_{w=0}^{W_{\max }}\left(P_{s}[m]\right) \cdot \pi(m, i, r, w)
\end{aligned}
$$

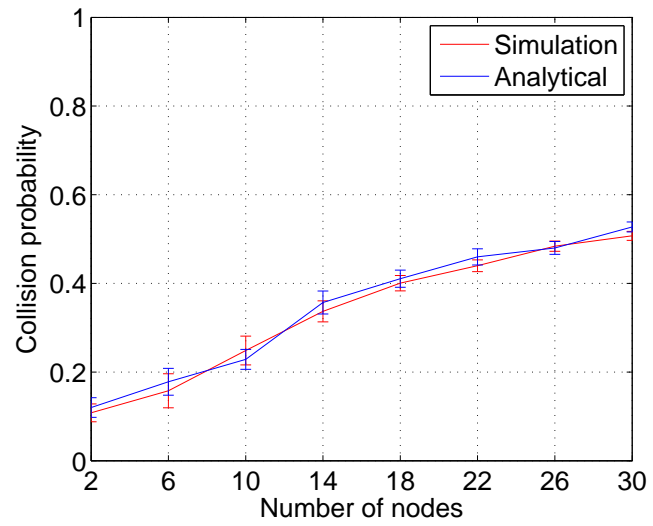

Fig. 4: Collision probability, $\left(N=30, L=10, \max _{R}=3\right)$

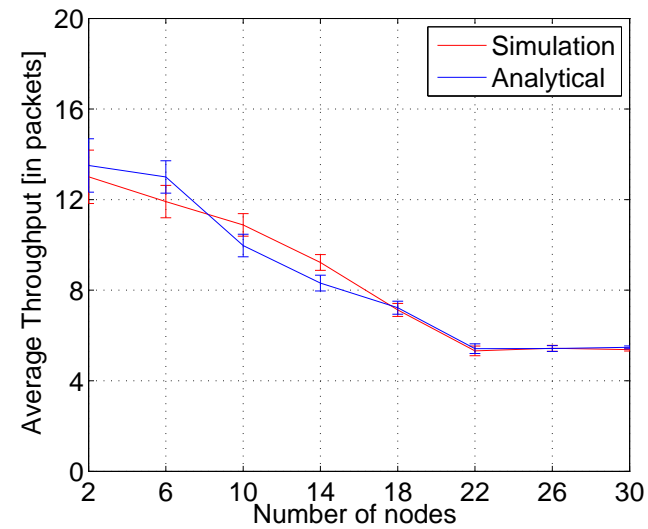

Fig. 5: Average Throughput, $\left(N=30, L=10, \max _{R}=3\right)$

We measure the network throughput when we increase the number of transmitters (Fig. 5). Increasing the number of nodes increases the number of collisions, and reduces both the network capacity and the reliability. Thus, most of the shared cells have to be statistically empty (with a larger duty cycle ratio) to provide an acceptable reliability.

\section{Delay}

We measured the delay as the time elapsed between the packet generation and its reception, derived from Little's theorem:

$$
\begin{aligned}
\text { Delay } & =\frac{\text { avg. } n b \text { pkts in queue }}{\text { avg nb. of arrivals }} \\
\text { avg. nb pkts in queue } & =\sum_{i=0}^{L} i \cdot \pi(i)
\end{aligned}
$$

The delay increases when more nodes are present (Fig. 6). Indeed, more collisions occur, and the packets have to enqueued longer before receiving an ack. In fact, when a packet faces a collision, it does not leave the queue in order to be retransmitted during another cell. The jitter increases similarly, making the performance less stable and predictive. 


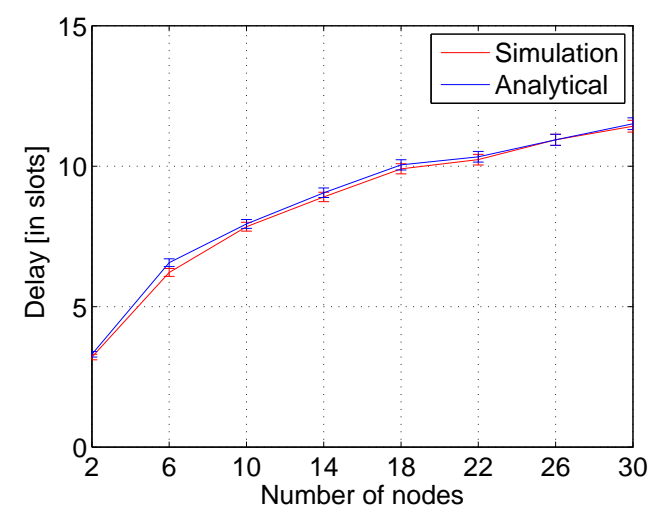

Fig. 6: Average delay, $\left(N=30, L=10, \max _{R}=3\right)$

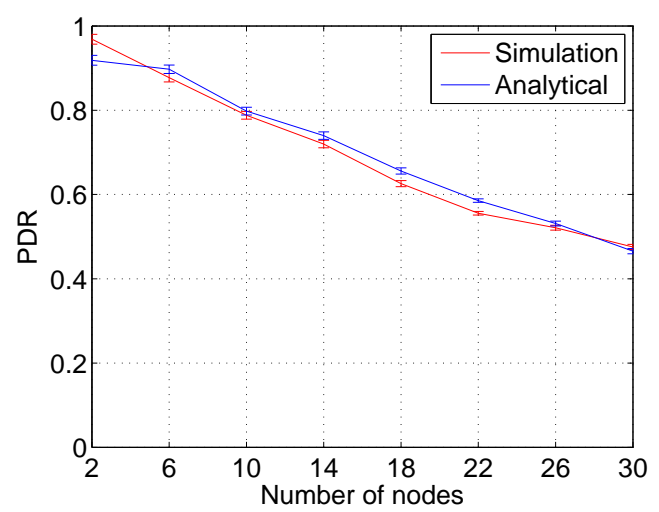

Fig. 7: Average L3-PDR, $\left(N=30, L=10, \max _{R}=3\right)$

\section{Network layer Packet Delivery Ratio}

We finally compute the PDR at the network layer to obtain the reliability provided to the application (Fig. 7). It corresponds to the ratio of acknowledged packets by the border router and the number of packets generated by the source node. For example a packet which is correctly acknowledged after $r$ retransmissions leads to a L3-PDR of $100 \%$. Thus:

$$
\begin{array}{r}
L 3-P D R=\frac{n b . \text { acks }}{n b \text { pkts txed }} \\
n b \text { acks. }=\sum_{m=0}^{N} \sum_{i=0}^{L} \sum_{r=0}^{\max _{R}} \sum_{w=0}^{W_{\max }}\left(P_{s}[m]\right) \cdot \pi(m, i, r, w)
\end{array}
$$

Without any surprise, the network layer PDR decreases when the number of contenders increase. However, it is still possible to provide a $60 \%$ PDR with 10 nodes by retransmitting several times the same packets. Thus, we can directly use our Markov Chain model in the scheduler so that it can decide the devices to group in a common shared cell, depending on the reliability required by the application.

\section{CONCLUSION \& Future Work}

We studied here analytically the behavior of TSCH when using shared cells to transmit a bursty traffic. Our model is based on Discrete Time Markov Chains, from which we derive the collision probability according to the number of contenders assigned to the same shared cell. In particular, we formulated the number of active nodes at any instant, according to a binomial distribution. TSCH is aggressive, and the number of collisions is very high in the shared cells, even with a medium traffic. However, link-layer retransmissions help to increase the end-to-end reliability.

In a future work, we plan to integrate this model in the scheduler, to derive the number of cells to assign to a group of contenders to respect a minimum end-to-end reliability. We also plan to integrate a location-dependent traffic model, where several sensors located in a given area detect the same event and generate correlated bursts of packets. The scheduler has consequently to consider this correlation when assigning the shared cells.

\section{REFERENCES}

[1] M. Wollschlaeger, T. Sauter, and J. Jasperneite. The Future of Industrial Communication: Automation Networks in the Era of the Internet of Things and Industry 4.0. IEEE Industrial Electronics Magazine, 11(1):17-27, March 2017.

[2] R. T. Hermeto, A. Gallais, and F. Theoleyre. Scheduling for IEEE802.15.4-TSCH and slow channel hopping MAC in low power industrial wireless networks: A survey. Computer Communications, 114:84 - 105, 2017.

[3] A. Elsts, X. Fafoutis, J. Pope, G. Oikonomou, R. J. Piechocki, and I. Craddock. Scheduling High Rate Unpredictable Traffic in IEEE 802.15.4 TSCH Networks. In International Conference on Distributed Computing in Sensor Systems(DCOSS). IEEE, 2017.

[4] Z. Dawy, W. Saad, A. Ghosh, J. G. Andrews, and E. Yaacoub. Toward Massive Machine Type Cellular Communications. IEEE Wireless Communications, 24(1):120-128, February 2017.

[5] J. Choi. Stability and Throughput of Random Access With CSBased MUD for MTC. IEEE Transactions on Vehicular Technology, 67(3):2607-2616, March 2018.

[6] C.-Y. Jung, H.-Y. Hwang, D.-K. Sung, and G.-U. Hwang. Enhanced Markov chain model and throughput analysis of the slotted CSMA/CA for IEEE 802.15. 4 under unsaturated traffic conditions. IEEE Transactions on Vehicular Technology, 58(1):473-478, 2009.

[7] C. Ouanteur, L. Bouallouche-Medjkoune, and D. Aïssani. An Enhanced Analytical Model and Performance Evaluation of the IEEE 802.15.4e TSCH CA. Wireless Personal Communications, 96(1):1355-1376, Sep 2017.

[8] T. Watteyne, A. Mehta, and K. Pister. Reliability Through Frequency Diversity: Why Channel Hopping Makes Sense. In PE-WASUN. ACM, 2009.

[9] IEEE Standard for Low-Rate Wireless Personal Area Networks (LRWPANs). IEEE Std 802.15.4-2015 (Revision of IEEE Std 802.15.42011), April 2016.

[10] G. Bianchi. Performance analysis of the IEEE 802.11 distributed coordination function. IEEE Journal on selected areas in communications, 18(3):535-547, 2000.

[11] I. Tinnirello, G. Bianchi, and Y. Xiao. Refinements on IEEE 802.11 distributed coordination function modeling approaches. IEEE Transactions on Vehicular Technology, 59(3):1055-1067, 2010.

[12] J. Misic, S. Shafi, and V. B. Misic. Performance of a beacon enabled IEEE 802.15. 4 cluster with downlink and uplink traffic. IEEE Transactions on Parallel and Distributed Systems, 17(4):361-376, 2006.

[13] D. De Guglielmo, B. Al Nahas, S. Duquennoy, T. Voigt, and G. Anastasi. Analysis and Experimental Evaluation of IEEE 802.15.4e TSCH CSMA-CA Algorithm. IEEE Transactions on Vehicular Technology, 66(2):1573-1588, Feb 2017. 